\title{
Mechanisms of TNF- $\alpha$ - and RANKL-mediated osteoclastogenesis and bone resorption in psoriatic arthritis
}

\author{
Christopher T. Ritchlin, ${ }^{1,2}$ Sally A. Haas-Smith,,${ }^{1,2}$ Ping Li, ${ }^{2}$ David G. Hicks, ${ }^{3}$ \\ and Edward M. Schwarz ${ }^{2}$ \\ ${ }^{1}$ Allergy, Immunology and Rheumatology Unit, and \\ ${ }^{2}$ The Center for Musculoskeletal Research, University of Rochester Medical Center, Rochester, New York, USA \\ ${ }^{3}$ Department of Pathology, The Cleveland Clinic, Cleveland, Ohio, USA
}

\begin{abstract}
Psoriatic arthritis (PsA) is an inflammatory joint disease characterized by extensive bone resorption. The mechanisms underlying this matrix loss have not been elucidated. We report here that blood samples from PsA patients, particularly those with bone erosions visible on plain radiographs, exhibit a marked increase in osteoclast precursors (OCPs) compared with those from healthy controls. Moreover, PsA PBMCs readily formed osteoclasts in vitro without exogenous receptor activator of NF- $\mathrm{KB}$ ligand (RANKL) or MCSF. Both osteoprotegerin (OPG) and anti-TNF antibodies inhibited osteoclast formation. Additionally, cultured PsA PBMCs spontaneously secreted higher levels of TNF- $\alpha$ than did healthy controls. In vivo, OCP frequency declined substantially in PsA patients following treatment with anti-TNF agents. Immunohistochemical analysis of subchondral bone and synovium revealed RANK-positive perivascular mononuclear cells and osteoclasts in PsA specimens. RANKL expression was dramatically upregulated in the synovial lining layer, while OPG immunostaining was restricted to the endothelium. These results suggest a model for understanding the pathogenesis of aggressive bone erosions in PsA. OCPs arise from TNF- $\alpha$-activated PBMCs that migrate to the inflamed synovium and subchondral bone, where they are exposed to unopposed RANKL and TNF- $\alpha$. This leads to osteoclastogenesis at the erosion front and in subchondral bone, resulting in a bidirectional assault on psoriatic bone.
\end{abstract}

J. Clin. Invest. 111:821-831 (2003). doi:10.1172/JCI200316069.

\section{Introduction}

Psoriatic arthritis (PsA) is an inflammatory joint disease that can be distinguished from rheumatoid arthritis (RA) on the basis of unique clinical features, the absence of rheumatoid factor, and characteristic radiographic findings (1). Patients frequently develop focal inflammation at multiple sites, including skin, joints, and tendon-insertion sites or entheses (2).

A notable propensity for aggressive bone erosions in PsA is well recognized (3) and is manifest radiographically as dramatic joint-space loss, large eccentric bone lesions, pencil-in-cup erosions, and acrolysis (extensive resorption of the distal phalanges) $(4,5)$. In PsA, periarticular bone mineralization is maintained and there

Received for publication June 4, 2002, and accepted in revised form January 28, 2003.

Address correspondence to: Christopher T. Ritchlin, Allergy, Immunology and Rheumatology Unit,

University of Rochester Medical Center, 601 Elmwood Avenue, Box 695, Rochester, New York 14642, USA.

Phone: (585) 275-2891; Fax: (585) 506-1979;

E-mail: Christopher_ritchlin@urmc.rochester.edu.

Conflict of interest: The authors have declared that no conflict of interest exists.

Nonstandard abbreviations used: psoriatic arthritis (PsA); rheumatoid arthritis (RA); osteoclast precursor (OCP); receptor activator of NF-KB (RANK); receptor activator of NF-KB ligand (RANKL); osteoprotegerin (OPG); osteoarthritis (OA). is often concomitant new bone formation in the form of periostitis and frank ankylosis, findings not seen in RA $(6,7)$. The presence of marked bone resorption coupled with adjacent new bone formation (often in the same digit) suggests a disordered pattern of bone remodeling in the psoriatic joint.

Osteoclasts, the principal cells responsible for bone resorption (8), are derived from mononuclear cell precursors of the monocyte/macrophage lineage (9). It has been proposed, based on experimental models, that pathological resorption is, at least in part, due to an increase in the number of these precursors (10). Indeed, elevated numbers of circulating osteoclast precursors (OCPs) have been identified in the peripheral blood of patients with aggressive multiple myeloma and the bone marrow of patients with Paget disease $(11,12)$. Thus, investigation of the factors that promote osteoclast development may provide insights into events responsible for pathologic bone loss in PsA.

Homeostatic differentiation of osteoclasts or osteoclastogenesis is a contact-dependent process directed by osteoblasts and stromal cells in the bone microenvironment $(13,14)$. Osteoblasts and stromal cells release two different signals that are necessary and sufficient for differentiation of OCPs into osteoclasts. The first, MCSF, binds the receptor c-fms, and the second, receptor activator of NF- $\mathrm{KB}$ ligand (RANKL), binds to RANK 
on the surface of OCPs (15). Since permissive quantities of MCSF are constitutively expressed in the bone microenvironment, it has been proposed that the relative expression of RANKL and its natural antagonist osteoprotegerin (OPG) ultimately controls osteoclastogenesis $(8,16-18)$. Furthermore, it has been demonstrated that minuscule quantities of RANKL are sufficient to synergize with TNF- $\alpha$ and potentiate osteoclastogenesis (13).

Our group has found that TNF- $\alpha$ directly increases the number of OCPs in mice genetically modified to overexpress TNF- $\alpha$ (hTNF-transgenic) and in normal mice injected with TNF- $\alpha$ (10). Additionally, treatment of hTNF-transgenic mice with anti-TNF agents reduces the number of OCPs to base line. In humans, TNF- $\alpha$ levels are elevated in the psoriatic synovium and joint fluid (19-21). In the present study, we analyzed OCP frequency in PsA patients and healthy controls. The role of TNF- $\alpha$ and RANKL in promoting osteoclast formation was also studied. We performed immunohistochemistry on synovial tissues and bone obtained from patients with PsA and osteoarthritis (OA) to determine expression patterns of RANKL, RANK, and OPG protein. The synovial expression of RANK, RANKL and OPG mRNA was also examined by RT-PCR. Lastly, the ability of TNF- $\alpha$ to modulate OCP frequency in vivo was examined by determination of the number of circulating OCPs in PsA patients before and after anti-TNF therapy.

\section{Methods}

Study population and treatment protocol. All clinical studies were carried out with the approval of the University of Rochester Medical Center Research Subjects Review Board and with informed consent. Synovium, cartilage, and bone specimens were obtained at the time of joint replacement surgery from five PsA, four RA, and four OA patients. PsA was diagnosed according to the Moll and Wright criteria (22), RA by the American College of Rheumatology criteria (23), and OA by physical examination and characteristic findings on plain $x$-ray. A blinded radiologist evaluated radiographs from PsA patients. Healthy controls had no acute or chronic joint pain and were in good health. None of the patients or controls was taking corticosteroids or second-line agents (methotrexate, gold, hydroxychloroquine, leflunomide, etanercept, or infliximab). Nine patients with erosive PsA were treated with anti-TNF agents: eight patients with $25 \mathrm{mg}$ etanercept twice per week and one patient with 5 $\mathrm{mg} / \mathrm{kg}$ infliximab at weeks $0,2,6$, and 14 .

OCPs from PBMCs. PBMCs were isolated from whole blood obtained from 24 PsA patients and 12 healthy controls. The PBMCs were separated on Ficoll gradients. Unfractionated PBMCs $\left(1 \times 10^{6}\right.$ cells per $\left.\mathrm{ml}\right)$ were placed in eight-well chamber slides containing $0.5 \mathrm{ml}$ $10 \%$ FCS-RPMI. Cultures were incubated in $6 \% \mathrm{CO}_{2}$ at $37^{\circ} \mathrm{C}$ for 14 days. Medium was replenished every 2-3 days. After 14 days in culture, slides were stained for tartrate-resistant acid phosphatase (TRAP; Sigma Diagnostics, St. Louis, Missouri, USA). Slides were viewed by light microscopy, and TRAP-positive cells with three or more nuclei were counted as osteoclasts. The scoring system that we used presents the data as the number of osteoclasts per $10^{6} \mathrm{PBMCs}$ recorded as the number of TRAP-positive multinuclear cells. This assay was performed because osteoclasts are derived exclusively from OCPs and there is currently no recognized surface marker for OCPs per se $(9,24)$. Cultures stimulated with $25 \mathrm{ng} / \mathrm{ml} \mathrm{MCSF}$ and $100 \mathrm{ng} / \mathrm{ml}$ RANKL served as positive controls.

The ability of these cells to resorb bone was demonstrated by culturing of PBMCs in $0.5 \mathrm{ml} 10 \%$ FBSRPMI on bovine bone wafers for 21 days. The cultured bone wafers, together with uncultured wafers, were stained with toluidine blue and photographed. The resorption area was quantified by density scan using Scion imaging software (Scion Corp., Frederick, Maryland, USA) after subtraction of the background in the uncultured wafers. These data are expressed as the percentage resorption area, calculated by dividing the total pitted area by the total surface area of the bone wafer.

Flow cytometry. PBMCs were prepared as described above, and the cells were centrifuged and resuspended in PBS containing 4\% FBS. Aliquots of $1 \times 10^{6}$ cells were incubated with anti-human CD11b (ICRF44), anti-human CD14 (M5E2), anti-human $\alpha_{v} \beta_{3}$ (CD51/ CD61) and related isotype controls (Pharmingen, San Diego, California, USA), or with fluorescein-conjugated RANKL (a gift from M. Tondravi, American Red Cross, Rockville, Maryland, USA). The cells were then washed with 4\% FBS-PBS. Data were acquired using a FACSCalibur instrument and analyzed by CellQuest software, version 3.1 (both from Becton Dickinson Immunocytometry Systems, Bedford, Massachusetts, USA).

Immunohistochemistry on synovial tissues, bone, and cartilage. All tissue samples were formalin-fixed, and bone specimens were decalcified in Immunocal (Decal Corp., Congers, New York, USA), dehydrated in a graded series of alcohols, and embedded in paraffin. Samples were cut in $3-\mu \mathrm{m}$ sections and mounted on glass slides. Sections were deparaffinized in xylene and rehydrated through a graded series of alcohols to distilled water. Endogenous peroxidase activity was quenched by $3 \%$ hydrogen peroxide. Antigen retrieval was performed in a pressure cooker (de-cloaking chamber; Biocare Medical, Walnut Creek, California, USA) using $0.01 \mathrm{M}$ citrate buffer. For OPG staining, citrate and glycerol buffer was used. Slides were blocked in 1:20 normal goat serum (Vector Laboratories Inc., Burlingame, California, USA). For OPG staining, normal horse serum was used as a blocking agent. Antibodies were diluted as noted below and incubated overnight at $4^{\circ} \mathrm{C}$. Following the incubation, slides were rinsed in PBS, and the biotin-conjugated secondary antibodies were applied for 30 minutes at room temperature. Slides were washed, and HRP-streptavidin (Zymed 
Table 1

Semiquantitative analysis of osteoclast numbers in PsA, RA, and OA

\begin{tabular}{ccc}
\hline & Tissue & Osteoclasts \\
PsA & Hip & $3+$ \\
& Knee & $2+$ \\
& Hip & $1+$ \\
& Foot & $1+$ \\
Knee & $3+$ \\
RA & Hip & $2+$ to $3+$ \\
& Knee & $3+$ \\
& Foot & $2+$ \\
OA & Knee & $1+$ \\
& Knee & 0 to $1+$ \\
& Hip & 0 to $1+$ \\
& Knee & 0 to $1+$ \\
& Knee & 0 to $1+$
\end{tabular}

Tissue samples, obtained at surgery, from five patients with PsA, four patients with RA, and four patients with OA were fixed, embedded, sectioned, and stained with $\mathrm{H} \& \mathrm{E}$. A pathologist blinded to the diagnosis semiquantitatively assessed the number of osteoclasts using the system described in Methods.

Laboratories Inc., South San Francisco, California, USA) was added at a 1:250 dilution in PBS for $30 \mathrm{~min}-$ utes at room temperature. Sections were washed once in PBS followed by deionized water, then incubated in AEC Chromagen (Romulin; AEC Biocare Medical, Walnut Creek, California, USA). Slides were counterstained with hematoxylin. Primary antibodies to RANK (rabbit anti-human Ab 1861) and RANKL (rabbit anti-human $\mathrm{Ab} 1862)$ were purchased from Chemicon International Inc. (Temecula, California, USA). RANK and RANKL antibodies were diluted in $2 \%$ normal goat serum in 1:20 BSA/PBS and applied at a 1:800 dilution. The secondary antibody, biotinylated goat anti-rabbit (Vector Laboratories Inc.), was added at a 1:200 dilution. OPG antibody (mouse anti-human mAb 805) purchased from R\&D Systems Inc. (Minneapolis, Minnesota, USA) was used at a dilution of 1:30. The secondary antibody, biotinylated horse anti-mouse (Vector Laboratories Inc.), was applied at a 1:200 dilution. Sections stained with only the secondary antibody served as a negative control. Slides were reviewed and scored by an independent pathologist blinded to the diagnosis. The osteoclast score was based on an assessment of the number of osteoclasts in Howship's resorption lacunae per $\times 20$ intermediate-power field in areas of active bone remodeling: $1+$, one to two osteoclasts per ten fields; $2+$, two to five osteoclasts per ten fields; $3+$, more than five osteoclasts per ten fields.

Analysis of RANKL, RANK, and OPG gene expression by $R T$-PCR. Synovium was obtained from six PsA and two OA patients undergoing total joint replacement or hand or foot surgery. RNA was isolated as previously described (25) and reverse-transcribed, and PCR was carried out under conditions described by Gravallese et al. (26) with custom primers from GIBCO BRL (Life Technologies Inc., Rockville, Maryland, USA). Primer sequences were RANKL sense, 5' CTATTTCAGAGCGCAGATGGAT-3'; RANKL antisense, 5'-TATGAGAACTTGGGATTTTGATGC-3' (26); RANK sense, 5' -TTAAGCCAGTGCTTCACGGG-3'; RANK antisense, 5'-ACGTAGACCACGATGATGTCGC-3' (27); OPG sense, $5^{\prime}$-GCTAACCTCACCTTCGAG-3'; OPG antisense, 5'-TGATTGGACCTGGTTACC-3' (28); GAPDH sense, 5' GCTCTCCAGAACATCATCCCTGCC-3'; GAPDH antisense, $5^{\prime}$-CGTTGTCATACCAGGAAATGAGCTT (26).

TNF- $\alpha$ ELISA. PBMC cultures were established in 24-well tissue-culture plates with cells from five PsA patients and five healthy controls. Unfractionated PBMCs were plated at $1 \times 10^{6}$ cells per $\mathrm{ml}$ in $10 \%$ FBSRPMI in $1 \mathrm{ml}$ total volume. The cells were incubated at $37^{\circ} \mathrm{C}$ and $6 \% \mathrm{CO}_{2}$ for 14 days. Culture supernatants were harvested and passed through a syringe filter to remove debris. Samples were stored at $-20^{\circ} \mathrm{C}$ until assayed. The assay was performed using matched antibody pairs against human TNF- $\alpha$ (Pierce Biotechnology, Rockford, Illinois, USA) following the manufacturer's technical-application procedure. Standards were serially diluted recombinant human TNF- $\alpha$ in culture media (Pierce Endogen). OD was recorded on a Bio-Rad microtiter plate reader (BioRad Laboratories, Hercules, California, USA). TNF- $\alpha$ is expressed as $\mathrm{pg} / \mathrm{ml}$.

Cocultures. PBMCs from PsA patients were cultured in 24-well tissue-culture plates at a density of $10 \times 10^{6}$ cells per $\mathrm{ml}$ in $1.0 \mathrm{ml}$ of $10 \%$ FBS-RPMI. Supernatants were harvested at day 14, filtered, and stored at $-20^{\circ} \mathrm{C}$. Healthy donor PBMCs isolated from three individuals were seeded in 96-well flat-bottomed culture plates at $2 \times 10^{5}$ cells per well with $50 \%$ PsA culture supernatant and 50\% normal media in $200 \mu \mathrm{l}$ total volume. In the initial experiments, supernatants from three different PsA PBMC cultures were added to PBMCs isolated from the three healthy controls. In subsequent experiments, supernatant from a PsA PBMC culture was added to PBMCs isolated from two different healthy controls. TNF- $\alpha$ activity was blocked by the addition of anti-TNF- $\alpha$ antibody (Pierce Endogen) at a final concentration of 2.5 $\mu \mathrm{g} / \mathrm{ml}$. The medium was replenished twice weekly after 14 days in culture; cells were stained for TRAP and osteoclasts were counted as described above.

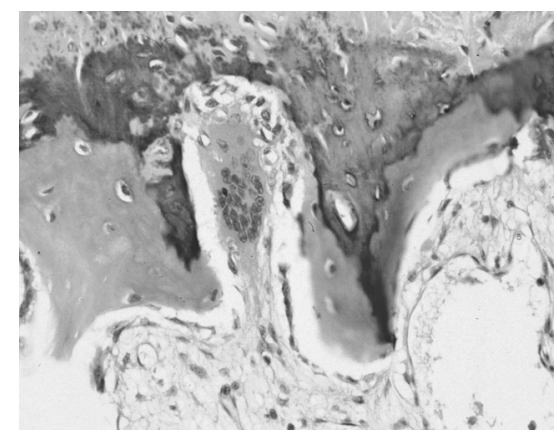

Figure 1

Osteoclasts are prominent in the psoriatic joint. A representative example of a large multinucleated osteoclast in Howship's lacuna is shown photographed with a $\times 40$ objective. 


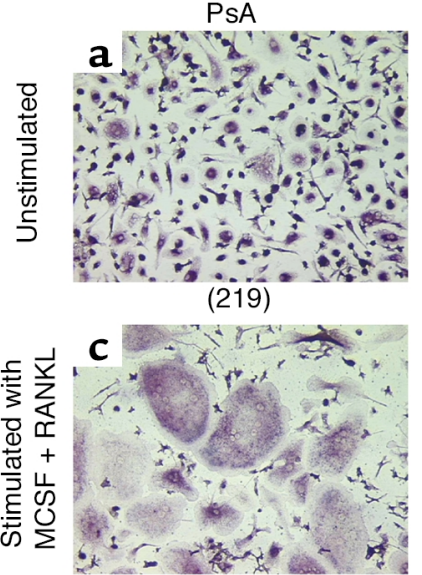

(1059)

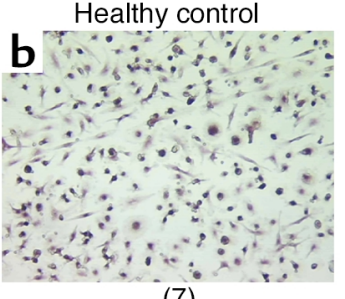

(7)

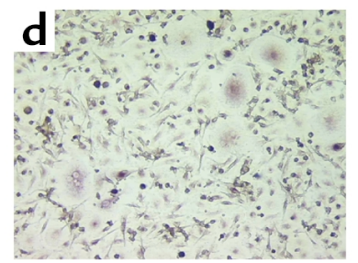

(264)

\section{Figure 2}

OCPs in PBMCs of PsA patients. PBMCs were obtained from PsA patients and healthy controls and cultured in the absence ( $\mathbf{a}$ and $\mathbf{b}$ ) or presence $(\mathbf{c}$ and $\mathbf{d})$ of MCSF $(25 \mathrm{ng} / \mathrm{ml})$ and RANKL $(100 \mathrm{ng} / \mathrm{ml})$ for 14 days, fixed and stained for TRAP, and photographed at $\times 10$ magnification. Numbers in parentheses represent the total number of osteoclasts per $10^{6}$ PBMCs for each sample.

Osteoclastogenesis inhibition by OPG. PBMC cultures were established from PsA donors as described above. OPG-Fc (R\&D Systems Inc.) was added at a final concentration of $1.0 \mu \mathrm{g} / \mathrm{ml}$. Etanercept (Amgen Inc., Thousand Oaks, California, USA) was also added to cultures as indicated at a final concentration of $1 \mu \mathrm{g} / \mathrm{ml}$. Cultures were maintained as previously described for 14 days prior to TRAP staining and osteoclast scoring.

Statistics. OCP data are expressed as the number of OCPs per $10^{6}$ PBMCs. Student's $t$ test of nonpaired data was used to analyze differences in OCP frequen$c y$, resorption area on bone wafers, expression of CD14 and CD11b, and supernatant TNF- $\alpha$ levels in PsA patients versus healthy controls. The number of PBMCs expressing CD14 in PsA patients before and after anti-TNF therapy, OCP numbers before and after supernatant stimulation, and blocking experiments with TNF and RANKL were analyzed by paired $t$ tests. The difference in the median number of OCPs in PsA patients with and without erosions was analyzed by the Mann-Whitney test.

\section{Results}

Osteoclasts were present in bone obtained from PsA patients. Although it is generally accepted that osteoclasts are the only cell type capable of bone resorption, these cells have not been characterized in the psoriatic joint. To formally document their role in this disease, initial studies were performed to ascertain whether osteoclasts were present at sites of focal erosion in PsA bone. Histology specimens from PsA, RA, and OA bone were examined and scored for osteoclast number as described in Methods. Table 1 shows that moderate to large numbers of osteoclasts were detected in bone samples from PsA patients. The majority of osteoclasts were found in resorption pits at the bone-pannus junction, or in cutting cones crossing the subchondral bone. Morphologically mature osteoclasts were not observed in the vascular lumen. Similarly, osteoclasts were increased in RA bone, while comparatively few were observed in the OA samples. In some PsA specimens, large osteoclasts with high nuclearity (more than 20 nuclei per cell) were observed (Figure 1).

Osteoclasts arise in unstimulated cultures of PBMCs from patients with PsA. Numerous multinucleated TRAP-positive cells were identified in low-density PBMC cultures from PsA patients without exogenous RANKL or MCSF (Figure 2a), while such cells were rare in PBMC cultures from healthy controls (Figure 2b). Addition of RANKL and MCSF to the cultures increased the size and number of osteoclasts in cultures from PsA patients (Figure 2c) and to a lesser degree in cultures from healthy controls (Figure 2d). To quantify this effect, PBMCs were isolated from 24 PsA patients and 12 healthy controls (Figure 3). The average number of circulating preosteoclasts in unstimulated cultures was significantly higher in PsA patients than in healthy controls (mean $168 \pm 39.9$ vs. $3.7 \pm 1.1$ osteoclasts per $10^{6}$ PBMCs; $\left.P<0.006\right)$. These results indicate that OCPs circulate in the peripheral blood of PsA patients in greater numbers than in healthy controls. Furthermore, these precursors progress to mature osteoclasts without exogenous RANKL and MCSF stimulation.

To determine whether the increased number of TRAPpositive multinucleated cells are derived from an increase in the number of mononuclear OCPs or from multinucleated inflammatory cells, we performed the following experiments. First, PBMCs isolated from seven PsA patients with erosive arthritis were allowed to settle overnight in eight-well chamber slides. The cells were fixed, TRAP-stained, and examined under a light microscope. We did not identify multinucleated or TRAP-positive cells in any of the patients. Second, using probes for surface markers of mononuclear OCPs, freshly isolated PBMCs from seven erosive PsA patients and seven healthy donors were stained for CD11b, CD14, CD51/CD61, and RANK and analyzed by FACS.

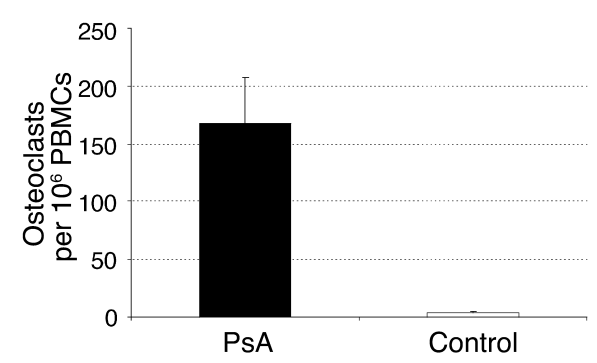

Figure 3

Large numbers of osteoclasts arise from unstimulated PsA PBMCs. PBMCs were obtained from 24 PsA patients and 12 healthy controls, cultured in the absence of MCSF and RANKL for 14 days, fixed, and stained for TRAP. The number of TRAP-positive multinucleated cells (osteoclasts) was counted and is presented as osteoclasts per million PBMCs plated. 


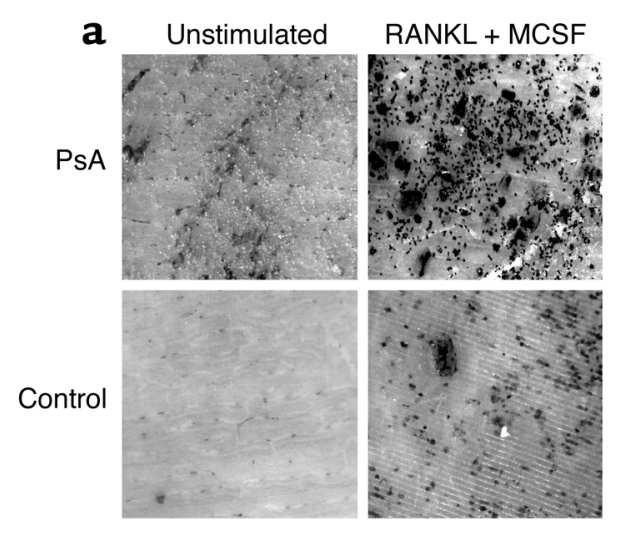

b

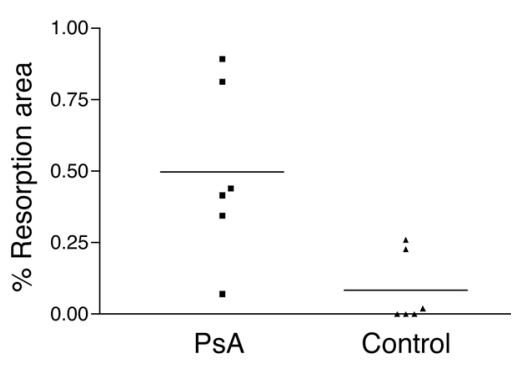

\section{Figure 4}

Cultured PsA PBMCs erode bone in a wafer assay. PBMCs were cultured with or without MCSF and RANKL on cortical bovine bone wafers. (a) After 21 days the wafers were stained with toluidine blue to identify resorption lacunae, shown photographed at $\times 10$. Representative PsA and control samples are shown. (b) The percentages of eroded surface area on the wafers from unstimulated cultures from PsA patients $(n=6)$ and healthy controls $(n=6)$ were quantified as described in Methods.
The percentage of PsA PBMCs expressing CD11b and CD14 was significantly greater than in healthy control PBMCs $(23.9 \% \pm 3.15 \%$ vs. $13.8 \% \pm 1.3 \%$; $P<0.006)$. Fur-

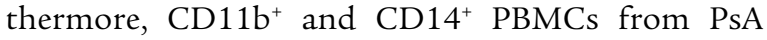
patients and controls also expressed CD51/CD61 and RANK, matching a phenotypic profile previously described for OCPs (24). In addition, we did not identify large or multinucleated cells in the forward and side scatter analysis.

To assess the bone-resorbing capacity of these cells, unstimulated PBMCs derived from PsA patients and healthy controls were cultured for 21 days on cortical bone wafers. Representative fields from stimulated and unstimulated bone wafer cultures are shown in Figure $4 \mathrm{a}$ along with the mean unstimulated values for all patients (Figure 4b). Cells from PsA patients $(n=6)$ eroded approximately seven times the surface area eroded by healthy controls $(n=6)$ (mean $0.49 \% \pm 0.31 \%$ vs. $0.08 \% \pm 0.12 \% ; P<0.009)$. These data demonstrate a functional osteoclast phenotype in cultured PsA PBMCs capable of enhanced bone-resorbing activity. This finding is consistent with the increased preosteoclast number detected in the PsA population.

Numbers of circulating preosteoclasts are bighest in patients with erosive arthritis. If OCP frequency contributes to inflammatory bone loss in PsA, one would predict that patients with erosions on plain radiographs would have higher numbers of circulating OPCs than PsA patients without erosions. Therefore, we analyzed OCP frequency in unstimulated PBMC cultures from ten PsA patients with and ten without bone erosions (Figure 5). PsA patients with one or more erosions on plain radiographs had a significantly greater number of OCPs than did PsA patients without erosions (median, 224 vs. 85 osteoclasts per $10^{6}$ PBMCs; $\left.P<0.002\right)$. These results suggest that OCPs contribute to osteolysis in PsA patients.

RANK, RANKL, and OPG expression in the PsA joint and bone. Based on our knowledge of the role of osteoclasts in mediating bone erosions in RA and the importance of RANK and RANKL signaling in the process, we investigated the expression pattern of RANK, RANKL, and OPG in PsA synovium and bone. Immunohistochemical analysis revealed that osteoclasts in resorption lacunae strongly expressed RANK. These osteoclasts were located at the synovial border of the pannus-bone interface (Figure 6 , $a$ and $b$ ) and in cutting cones in the subchondral bone (Figure 6c). In addition, RANK-positive mononuclear cells were detected adjacent to blood vessels traversing the synovium (Figure 6, a and b) and around vessels located in the subchondral bone (Figure 6d). The staining patterns of RANKL and OPG in the synovium are illustrated in Figure 7 . Intense RANKL immunoreactivity was present throughout the synovial lining layer (Figure 7a), while OPG staining (Figure $7 \mathrm{~b}$ ) was restricted to endothelial cells beneath the synovial lining, away from sites of active erosion. Tissue architecture can be determined in $\mathrm{H} \& \mathrm{E}$-stained sections (Figure $7 \mathrm{~d}$ ), while specific staining is not seen in the negative control (Figure 7c). RANK and RANKL staining was weak to absent in all OA tissues examined (data not shown).

To confirm the immunohistochemical studies, RT-PCR was performed on synovial membranes to analyze the pattern of RANK, RANKL, and OPG expression in tissues isolated from six patients with PsA and two with OA (Figure 8). Five patients with erosive PsA expressed RANKL mRNA in synovial tissues; however, the sixth PsA patient, who did not have bone erosions, did not express RANKL message.

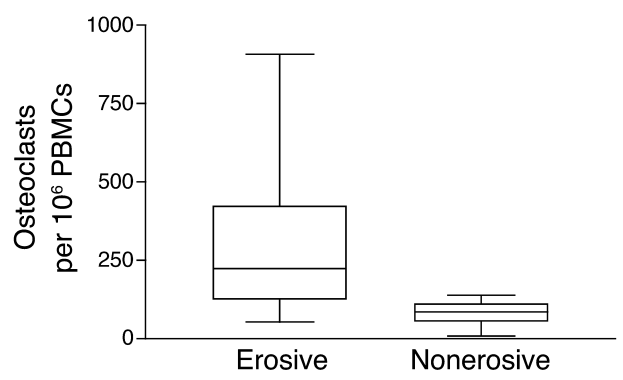

\section{Figure 5}

OCP frequency in peripheral blood is greater in PsA patients with erosive arthritis. PBMCs were obtained from ten PsA patients with and ten PsA patients without erosions on plain radiographs. The cells were cultured, fixed, and stained for TRAP, and osteoclast numbers were determined as described in Methods. Data are expressed as median osteoclasts per $10^{6}$ PBMCs. 

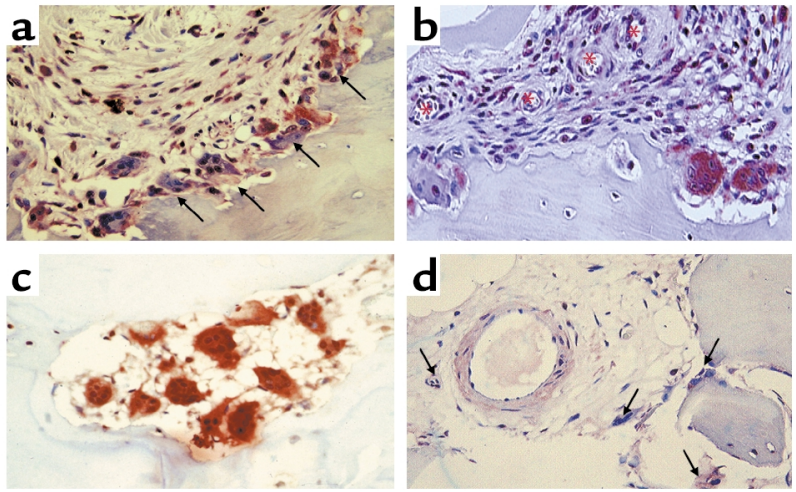

\section{Figure 6}

RANK expression in the psoriatic joint. Tissues from PsA patients containing synovium and bone were obtained following surgery and processed for immunohistochemistry as described in Methods. Antibodies specific for RANK stain red-brown. RANK-positive osteoclasts (arrows) are prominently located at the synovial edge of the pannusbone interface, shown photographed at $\times 20$ (a), and RANK-positive mononuclear cells increase in number moving from the endothelium (asterisks) to the erosion front $(\times 20)(\mathbf{b})$. In subchondral bone, RANK-positive osteoclasts were observed in cutting cones that are void of other cell types, as shown photographed at $\times 20$ magnification (c). RANK-positive mononuclear cells and osteoclasts (arrows) surround an endothelial cell traversing subchondral bone (d).

The finding of RANK expression in three of the six PsA synovial samples further supports the immunohistochemical data. Five of six PsA and one of two OA tissues expressed OPG.

Anti-TNF therapy reduces OCP frequency in PsA. It has been previously demonstrated that TNF- $\alpha$ is elevated in psoriatic synovium and synovial fluid (18-20). Furthermore, TNF- $\alpha$ can enhance osteoclastogenesis in the presence of minuscule amounts of RANKL (13). To delineate the effects of TNF- $\alpha$ on OCP frequency in vivo, we studied five patients with erosive PsA treated with anti-TNF agents (four with etanercept and one with infliximab). The number of OCPs was measured before and 12 weeks after initiation of therapy (Figure 9a). Each of the patients experienced a decrease in the number of tender and swollen joints and improved physician and patient global assessment. There was also a significant reduction $(79-96 \% ; P<0.001)$ in the number of OCPs following anti-TNF therapy in all patients. In addition, we observed a consistent decrease in the percentage of $\mathrm{CD} 11 \mathrm{~b}^{+} \mathrm{CD} 14^{+} \mathrm{PBMCs}$ (Figure 9, $b-d)$. These results strongly suggest that TNF- $\alpha$ directly contributes to the increased OCP frequency observed in PsA patients.

Release of biologically active TNF- $\alpha$ by PSA PBMCs. To determine whether the increase in the number of OCPs is the result of elevated TNF- $\alpha$ in PsA patients, the amount of TNF- $\alpha$ released by unfractionated PBMCs cultured without MCSF and RANKL from five PsA patients and five healthy controls was analyzed $(198.6 \pm 86.07 \mathrm{pg} / \mathrm{ml}$ vs. $25.8 \pm 13.40 \mathrm{pg} / \mathrm{ml}$, respectively; $P<0.04)$. In parallel experiments, PsA culture supernatants harvested from three PsA patients with high TNF- $\alpha$ levels stimulated increased osteoclast formation when added in vitro to PBMCs from healthy donors $(n=3)$. As shown in Figure 10a, unstimulated PBMCs from healthy controls yielded few osteoclasts. The number of osteoclasts increased following addition of each of the three PsA supernatants. Two of three supernatants significantly increased osteoclast numbers in the healthy control PBMC cultures. In subsequent experiments, the addition of anti-TNF- $\alpha$ antibody blocked supernatant-induced osteoclastogenesis (Figure 10b). Two healthy controls, different from those in Figure 10a, showed an increase in number of osteoclasts following addition of PsA supernatant 2 from Figure 10a. This increase was inhibited by addition of anti-TNF antibodies. These experiments suggest that PBMCs from PsA patients secrete significantly greater quantities of biologically active TNF- $\alpha$ than PBMCs from healthy controls.

OPG inhibits osteoclastogenesis in unstimulated PsA $P B M C$ s. Previous studies have convincingly established that RANKL is an essential factor in the promotion of osteoclast development in the inflamed joint (29-31). To examine the impact of RANKL on osteoclast formation in PsA, unstimulated PsA PBMCs were cultured in the presence of OPG (Figure 11). The mean number of TRAP-positive multinuclear cells in unstimulated cultures was $45 \pm 5$ per $10^{6}$ PBMCs. This number significantly declined to $14 \pm 4$ in the presence of $1.0 \mu \mathrm{g} / \mathrm{ml}$ of OPG. Since it is known that TNF- $\alpha$ strongly synergizes with trace amounts of RANKL $(13,32,33)$, we tested the combination of OPG and etanercept, which further suppressed osteoclast formation to $8 \pm 2$ osteoclasts per $10^{6}$ PBMCs.
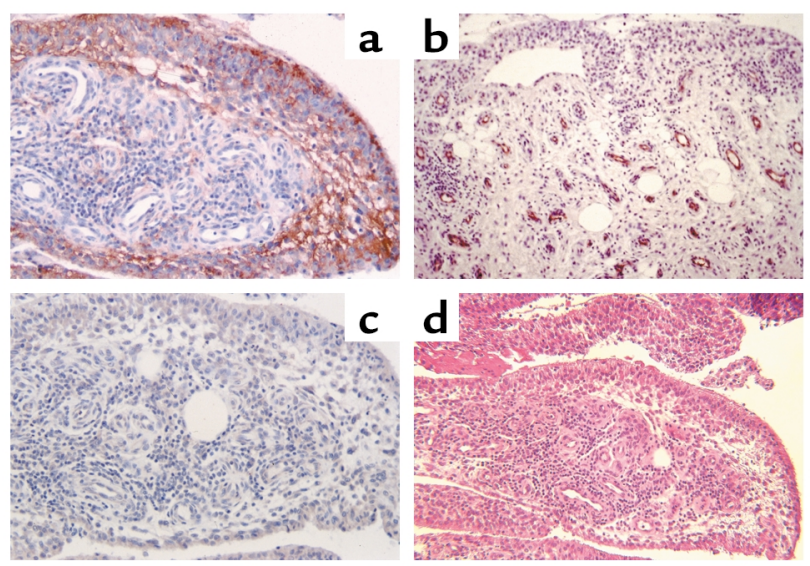

\section{Figure 7}

RANKL and OPG expression in the PsA synovium. Retrieval tissues containing synovium from PsA patients were processed for immunohistochemistry with antibodies specific for RANKL and OPG as described in Methods. A representative synovial membrane from a PsA patient stained with anti-RANKL antibody (a), anti-OPG antibody (b), secondary antibody only (c), and $\mathrm{H} \& \mathrm{E}(\mathbf{d})$ are shown photographed at $\times 20$. Of note is the very specific and intense RANKL staining by synovial lining cells and the OPG staining restricted to the endothelial cells below the synovial lining. 


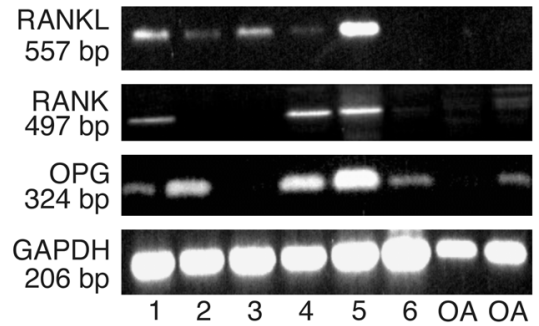

Figure 8

RANK, RANKL, and OPG expression in PsA synovium. Total RNA was isolated from PsA and OA synovium and used as the template to determine RANK, RANKL, and OPG mRNA expression by RT-PCR as described in Methods. The sizes of the specific PCR products are indicated. Lanes 1-5 show PsA patients with radiographic bone erosions, lane 6 shows a patient without erosions, and lanes 7 and 8 show OA patients.

The marked reduction in osteoclast formation in cultures incubated with OPG supports the concept that RANKL expression is a critical event in the promotion of osteoclastogenesis in the psoriatic joint.

\section{Discussion}

In psoriatic arthritis (PsA), bone erosions can be extensive, resulting in joint deformity and disability. These erosions differ markedly from the periarticular osteopenia and pericapsular bone loss commonly observed in rheumatoid joints (34). While these radiographic features suggest a different mechanism of bone loss in PsA, understanding of the basis of this difference has been impeded because the events that lead to psoriatic bone resorption have not been well defined. To elucidate this process, we sought to clarify how OCPs and the regulatory molecules RANK, RANKL, and OPG may orchestrate osteolysis in PsA.

Our results demonstrate that osteoclasts are prominently situated at the bone-pannus junction and in cutting cones traversing the subchondral bone in the psoriatic joint. In addition, OCPs are markedly increased in the circulation of PsA patients, most strikingly in those with bone erosions on plain radiographs. These cells express the surface markers CD11b, CD14, CD51/CD61, and RANK. A pivotal role for TNF- $\alpha$ in promoting OCP formation is supported by the observations that blocking TNF- $\alpha$ in vivo markedly suppressed the number of circulating OCPs and that cultured PsA PBMCs spontaneously release high quantities of biologically active TNF- $\alpha$. Immunohistochemical studies delineated the presence of RANK-positive cells in synovium and adjacent to blood vessels in subchondral bone. Furthermore, synovial lining cells stained strongly for RANKL, while OPG expression was confined to the endothelium. These data suggest that OCPs enter a synovial microenvironment characterized by a high ratio of RANKL to OPG expression, facilitating osteoclastogenesis and bone resorption.

To our knowledge, this is the first study demonstrating the presence of increased numbers of circulating OCPs in patients with inflammatory arthritis.
The initial impetus for the concept of an expanded pool of OCPs arose from studies on patients with Paget disease and multiple myeloma. Examination of bone marrow cultures from Paget patients revealed an increase in the number of committed OCPs compared with that in healthy controls (12). Similarly, PBMCs cultured from patients with multiple myeloma and bone lesions, but not those without bony involvement or healthy controls, gave rise to osteoclasts that resorbed bone in vitro when cultured in the presence of a murine stromal line (11). Faust et al. extended these observations by showing that osteoclasts can develop from unstimulated PBMCs derived from healthy controls when grown at high density; however, the number of osteoclasts was not quantified, and they demonstrated weak bone resorption properties (35). In pilot studies, we noted that numerous osteoclasts were present in unstimulated wells of PBMCs cultured from PsA patients, even when the cultures were seeded at low density. Thus, we modified our experimental protocol, analyzing OCP frequency at low density in the absence of exogenous factors such as RANKL and MCSF. Using this approach, osteoclasts were identified by positive TRAP staining and multinuclearity. These cells were shown to be functional by their ability to form pits on bone wafers. Compared with healthy controls, PsA patients had markedly more OCPs, and these cells resorbed significantly greater quantities of bone. It should be noted that the difference in resorption area between control and PsA patients was less than the
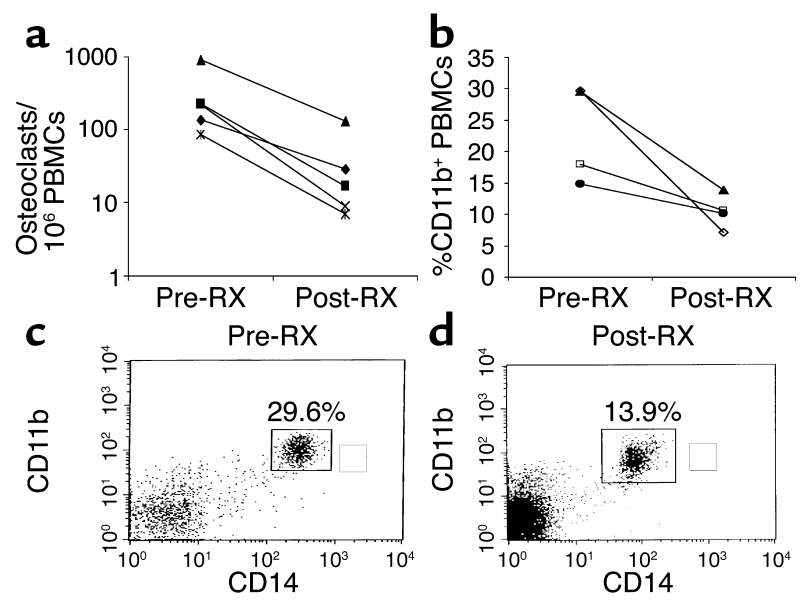

Figure 9

Anti-TNF- $\alpha$ therapy reduces OCP frequency in patients with PsA. (a) PBMCs from five PsA patients were cultured to determine the OCP frequency before and after 12 weeks of anti-TNF- $\alpha$ therapy (four patients with etanercept and one with infliximab). The data are expressed as osteoclasts per $10^{6}$ PBMCs. The number of OCPs in peripheral blood was significantly reduced in all of the patients $(P<0.001)$. (b) The percentage of CD11b+ PBMCs significantly declined in four patients with erosive PsA, following 2 weeks of etanercept therapy, as determined by FACS $(P<0.026)$. (c and d) Representative histograms of CD14/CD11b staining from a PsA patient before (c) and after (d) etanercept therapy. 


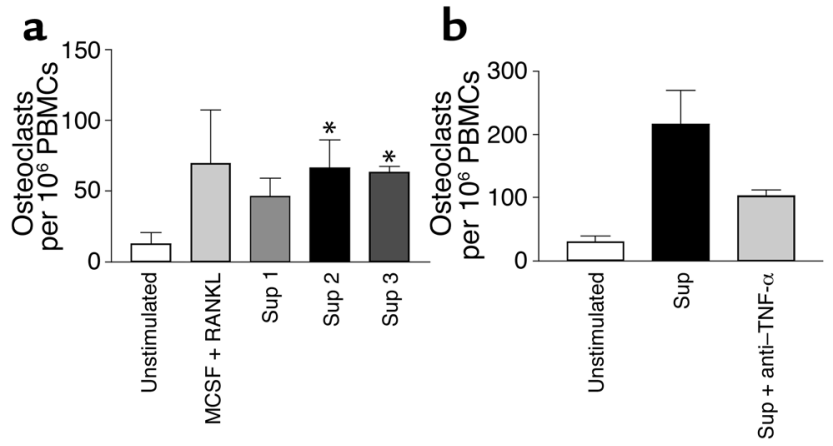

Figure 10

TNF- $\alpha$ produced by PsA PBMCs enhances osteoclastogenesis. (a) Supernatants from cultured PsA PBMCs were added to PBMCs from healthy donors to stimulate osteoclastogenesis as described in Methods. The mean number of osteoclasts that arose from three unstimulated healthy control PBMC cultures (white bar), cultures incubated with MCSF and RANKL (light gray bar), and cultures to which PsA and PBMC supernatants (black, medium gray, and dark gray bars) were added are presented as the mean $\pm \mathrm{SD}\left({ }^{*} P<0.02\right)$. (b) To determine the effect of TNF- $\alpha$ in the cocultures, PsA PBMC supernatant 2 (Sup 2 ) from a was added to two different healthy control PBMC cultures, and osteoclasts were counted with or without anti-TNF antibodies.

difference in OCP frequency between the two groups. Potential explanations include the inability to measure the depth of resorption pits, species differences (human PBMCs on bovine bone), and the fact that there is no literature demonstrating a correlation between the number of OCPs and in vitro measures of bone loss. The additional finding that the increase in OCP frequency correlated with clinical erosions indicates that the size of the precursor pool may be a dependent factor that contributes directly to bone resorption in PsA.

Studies in mice demonstrated that systemic TNF- $\alpha$ directly increases OCP frequency and that this elevation is reversible by anti-TNF therapy (10). Here we show that this is also true in PsA, as increased OCP frequency declined significantly in five of five patients treated with anti-TNF therapy, which paralleled clinical improvement. Moreover, PsA PBMCs spontaneously released high levels of TNF- $\alpha$ in vitro. TNF- $\alpha$ secreted by these cells promoted osteoclastogenesis that was blocked with anti-TNF- $\alpha$ antibodies. Blocking RANKL with OPG also substantially decreased the number of OCPs that arose from PsA PBMCs. Thus, our results imply that TNF- $\alpha$ triggers a systemic increase in the number of circulating OCPs and suggest that this may be a critical event in the modulation of psoriatic bone resorption. While these data strongly support the concept that TNF- $\alpha$ released by PsA PBMCs promotes an increased OCP frequency, they do not establish these cells as the principal source of the TNF- $\alpha$. Further studies designed to specifically address this question are required.

Of particular relevance to the observations outlined above are data demonstrating that TNF- $\alpha$ is a pivotal cytokine in PsA. TNF has been isolated from psoriatic synovial fluid, and psoriatic synovial explants release elevated levels of TNF- $\alpha$, which were highest in patients with erosive arthritis $(19,20)$. Also, psoriatic synovial lining cells express TNF- $\alpha$ protein (21). Perhaps the most convincing evidence stems from clinical trials showing that TNF blockade dramatically ameliorates psoriatic joint pain and swelling; this evidence led to the Food and Drug Administration's first approval of a drug, etanercept, for treatment of PsA $(36,37)$. Lastly, in a recent report, TNF inhibition improved clinical parameters of arthritis and reversed abnormal MRI bone and soft tissue signals in spondyloarthropathy patients with active joint and entheseal inflammation (38).

Although the precise phenotype of the precursor cell was not directly addressed in these experiments, we did find that PBMCs express CD11b, CD14, CD51/CD61, and RANK, established markers of mononuclear OCPs (14). It has been shown that approximately $2 \%$ of PBMCs can be stimulated to give rise to osteoclasts in vitro $(39,40)$. Interestingly, CD14 $4^{+}$monocytes can also differentiate into dendritic cells and macrophages (9, 41). Presumably, events in the bone marrow, circulation, and possibly the synovium determine the fate of a particular monocyte. Indeed, following exposure to RANKL and MCSF, a subpopulation of monocytes rapidly loses the CD14 marker and acquires an osteoclast phenotype (42), underscoring the critical importance of the RANK signaling pathway in osteoclastogenesis.

The discovery of RANK, RANKL, and OPG as the final effector molecules ultimately regulating osteoclastogenesis and bone resorption has provided a fundamental insight into the mechanisms of osteolysis in metabolic bone diseases $(8,43,44)$. Definitive proof in support of this paradigm has also been provided in animal models of inflammatory arthritis (10,39-41). In RA, investigators found that RANKL mRNA is expressed by $\mathrm{T}$ lymphocytes and synoviocytes isolated from lining membranes $(26,45,46)$. It has also been demonstrated that fibroblast-like synoviocytes can induce osteoclastogenesis when cocultured with

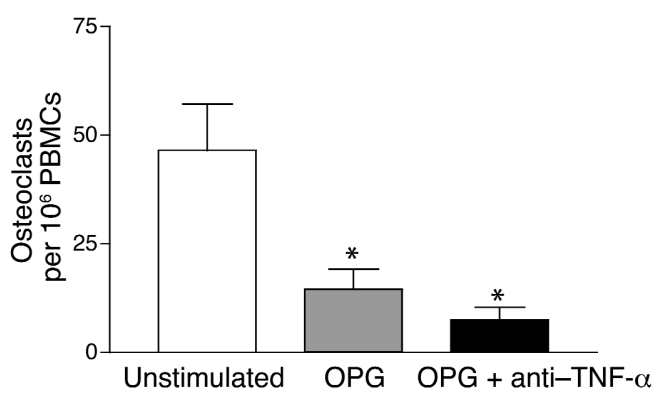

\section{Figure 11}

OPG inhibits osteoclast formation in unstimulated PsA PBMCs. TRAP osteoclastogenesis assays were performed on PsA PBMCs cultured without RANKL or MCSF in the continuous presence of OPG-Fc (1 $\mu \mathrm{g} / \mathrm{ml})$. Etanercept $(1 \mu \mathrm{g} / \mathrm{ml})$ was added as indicated. One representative experiment out of three is shown. Data are expressed as the mean \pm SEM of four independent wells $\left({ }^{*} P<0.05\right)$. 


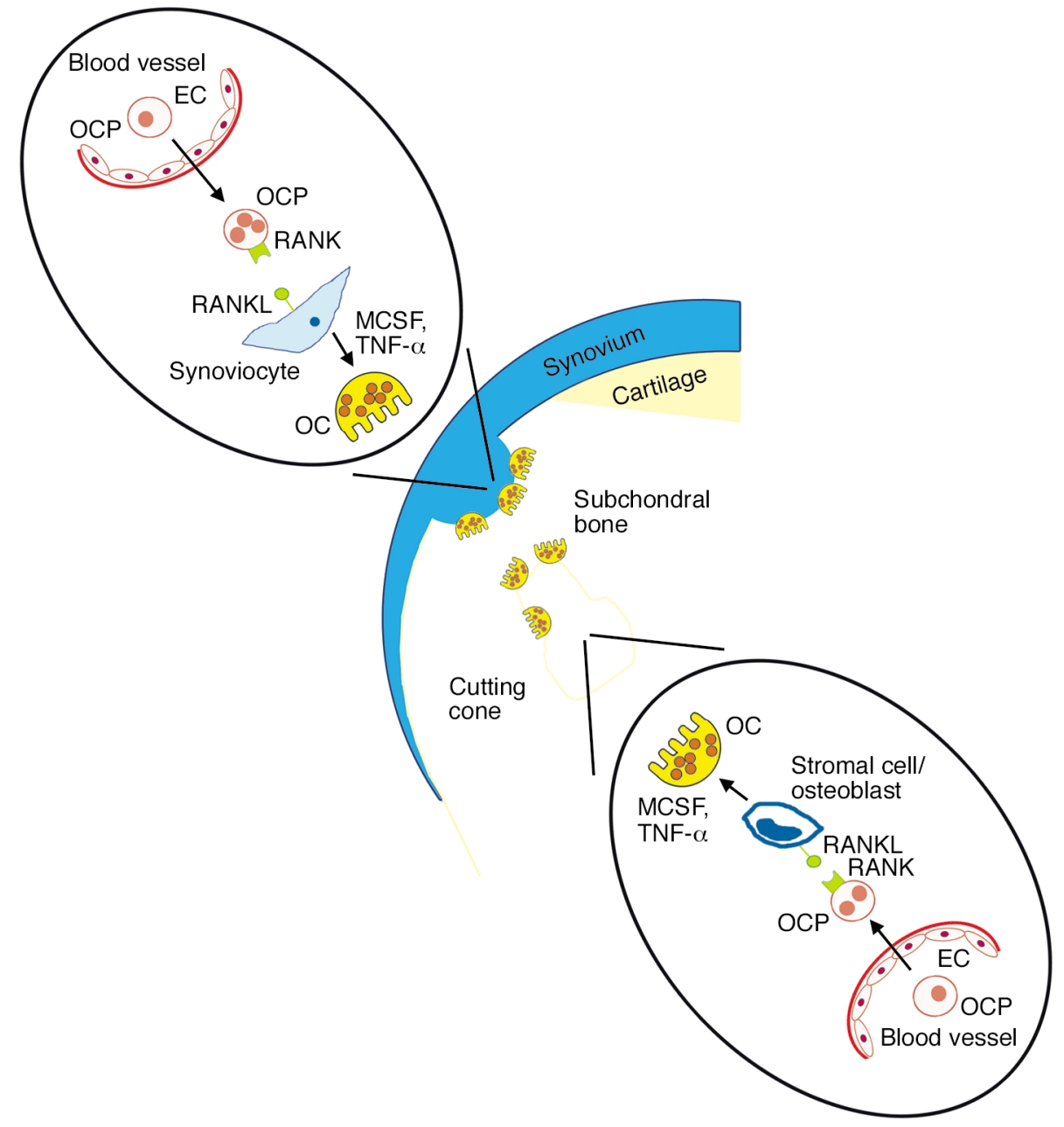

\section{Figure 12}

Schematic model of osteolysis in the psoriatic joint. Extensive erosions observed in the PsA joint are mediated by a bidirectional attack on bone. We propose that circulating OCPs enter the synovium and are induced to become osteoclasts by RANKL expressed by synoviocytes (outside-in). In parallel, OCPs traverse endothelial cells in the subchondral bone and undergo osteoclastogenesis following RANKL stimulation from osteoblasts and stromal cells (inside-out).
PBMCs (47). In our immunohistochemistry experiments, we found that PsA synovial lining cells stained intensely for RANKL, a finding not observed in OA synovial tissues. The RANKL appeared to be relatively unopposed by OPG, since staining for this molecule was restricted to the endothelium. The likely targets for this synovial cell RANKL are the perivascular RANK-positive mononuclear cells in the synovium and subchondral bone. The finding of RANK-positive mononuclear cells in the synovium, confirmed by elevated RANK mRNA expression in at least some of our PsA patients, was in line with previous studies that detected TRAP-positive cells in RA synovium and report that osteoclasts can be generated from RA synovium and bone $(33,48,49)$. We observed a gradient of RANK staining by mononuclear cells that increased in intensity from the perivascular region in the subsynovium to the erosion front, where synoviocytes and osteoclasts exhibited the strongest RANK expression. We speculate that this gradient is directed by the elevated RANKL and TNF- $\alpha$ expressed by PsA synoviocytes. Ultimately, RANKL stimulation of these precursor cells could result in the genesis of RANK-positive multinucleated osteoclasts that directly erode the bone matrix. Support for the critical role of RANKL is provided by our experiments indicating that OPG significantly blocked osteoclast formation in PsA PBMCs.

A central question that remains to be addressed regards the specificity of these findings to PsA. As previously discussed, osteoclasts have been detected in rheumatoid synovium $(48,50)$. Furthermore, while osteoclast numbers in PsA tissues were considerably greater than in OA samples, they were not significantly different from those in RA samples. Clearly, larger sample sizes are required to determine whether the number of osteoclasts differs in RA and PsA; but, assuming that it does not, what mechanisms could account for the aggressive bone resorption observed in many PsA patients? First, the number of circulating OCPs may be higher in PsA, resulting in a more sustained assault on bone. Second, the ratio of RANKL to OPG may be significantly greater in patients with destructive PsA, or, alternatively, levels of antiosteoclastogenic factors such as IFN- $\gamma$, IL-12, or GM-CSF could be higher in the rheumatoid joint. Third, the striking increased vascularity and vessel tortuosity characteristic of PsA but not RA (51) may facilitate enhanced recruitment and entry of OCPs into the joint. Finally, other pro-osteoclastogenic factors such as IL-1 
may be present in greater quantities in PsA joints, providing an additional osteoclast activation signal. In support of this latter mechanism is the observation that IL-1 was markedly elevated in psoriatic but not rheumatoid synovial explants obtained from patients with erosive joint disease (19).

Taken together with the established literature, the results of this study lead us to propose a mechanism for the destructive pathology observed in many psoriatic joints (Figure 12). In this model, TNF- $\alpha$ increases the number of circulating OCPs in PsA patients. In the case of "outside-in" erosion, OCPs enter a highly vascular psoriatic synovial membrane containing tortuous blood vessels and adhere to activated endothelial cells that have been stimulated by proinflammatory cytokines (52). Exposure to TNF- $\alpha$ could induce the expression of fibronectin and vitronectin receptors on endothelial cells, as described by McGowan et al., facilitating OCP binding and tissue migration (53). Simultaneously, the high level of OPG expressed by the endothelial cells would suppress osteoclastogenesis, permitting smaller undifferentiated OCPs to migrate through the dense pannus and target bone at a significant distance from the vessel. Upon arrival at the bone-pannus junction, OCPs bind RANKL on the surface of synoviocytes and, in the presence of TNF- $\alpha$ and MCSF, undergo osteoclastogenesis and erode bone. In the case of "outside-in" resorption, OCPs enter the subchondral environment in vessels that are in immediate proximity to bone. Following translocation through the endothelium, it is conceivable that OCPs are exposed to TNF- $\alpha$-induced RANKL on the surface of osteoblasts and stromal cells $(52,54)$, resulting in the generation of osteoclasts that line cutting cones devoid of synovial tissue. In this scenario, mature osteoclasts mount a bidirectional assault, resorbing bone matrix in the subchondral bone and at the pannus-bone interface. Thus, there are two critical steps in the osteolytic pathway mediated by TNF- $\alpha$ : increase in the frequency of circulating OCPs, and upregulation of RANKL expression in the joint. In this model, patients with generalized inflammatory disease (Crohn disease, psoriasis) may have an expansion of $\mathrm{CD} 14^{+} \mathrm{CD} 11 \mathrm{~b}^{+}$cells that differentiate into dendritic cells or macrophages, but not osteoclasts. In view of the reported findings, antagonism of TNF- $\alpha$ may prove to be an effective strategy for inhibiting bone destruction in PsA.

\section{Acknowledgments}

This project was funded by National Institute of Arthritis and Musculoskeletal and Skin Diseases grant AR-47186-01 and a Howard Hughes Pilot Grant. The authors wish to thank Barbara Stroyer for her expertise in immunohistochemistry; Robert Durham, clinical studies coordinator and Tianmeng Shao, research associate, for their assistance in recruiting subjects for this study; and John P. Leddy for critically reviewing the manuscript.
1. Winchester, R. 1993. Psoriatic arthritis. In Dermatology in general medicine. T.B. Fitzpatrick, A.Z. Eisen, K. Wolff, F.M. Freeberg, and K.F. Austin, editors. McGraw-Hill. New York, New York, USA. 515-527.

2. Helliwell, P., Marchesoni, A., Peters, M., Barker, M., and Wright, V. 1991. A re-evaluation of the osteoarticular manifestations of psoriasis. Br. J. Rheumatol. 30:339-345.

3. Gladman, D.D. 1998. Psoriatic arthritis. Rheum. Dis. Clin. North Am. 24:829-844.

4. Resnick, D., and Niwayama, G. 1981. Psoriatic arthritis. In Diagnosis of bone and joint disorders. D. Resnick and G. Niwayama, editors. W.B. Saunders Co. Philadelphia, Pennsylvania, USA. 1103-1109.

5. Resnick, D., and Niwayama, G. 1989. Psoriatic arthritis. In Bone and joint imaging. D. Resnick, editor. W.B. Saunders Co. Philadelphia, Pennsylvania, USA. 320-328.

6. Resnick, D., and Niwayama, G. 1977. On the nature and significance of bony proliferation in "rheumatoid variant" disorders. AJR Am. J. Roentgenol. 129:275-278.

7. Bywaters, E.G., and Dixon, A.S. 1965. Paravertebral ossification in psoriatic arthritis. Ann. Rheum. Dis. 24:313-331.

8. Teitelbaum, S.L. 2000. Bone resorption by osteoclasts. Science. 289:1504-1508.

9. Massey, H.M., and Flanagan, A.M. 1999. Human osteoclasts derive from CD14-positive monocytes. Br. J. Haematol. 106:167-170.

10. Li, P., Schwarz, E.M., O’Keefe, R.J., Boyce, B.F., and Xing, L. 2002. Systemic TNF $\alpha$ promotes erosive bone resorption by increasing the number of $\mathrm{CD} 11 \mathrm{~b}^{+}$osteoclast progenitors in the periphery which are dependent on RANK signaling for osteoclastogenesis. J. Bone Miner. Res. 17S(Suppl.):130. (Abstr.)

11. Gregoretti, M.G., et al. 1995. Osteoclast precursors circulate in the peripheral blood of patients with aggressive multiple myeloma. Leukemia. 9:1392-1397.

12. Demulder, A., Takahashi, S., Singer, F.R., Hosking, D.J., and Roodman, G.D. 1993. Abnormalities in osteoclast precursors and marrow accessory cells in Paget's disease. Endocrinology. 133:1978-1982.

13. Lam, J., et al. 2000. TNF- $\alpha$ induces osteoclastogenesis by direct stimulation of macrophages exposed to permissive levels of RANK ligand. J. Clin. Invest. 106:1481-1488.

14. Suda, T., et al. 1999. Modulation of osteoclast differentiation and function by the new members of the tumor necrosis factor receptor and ligand families. Endocr. Rev. 20:345-357.

15. Lacey, D.L., et al. 1998. Osteoprotegerin ligand is a cytokine that regulates osteoclast differentiation and activation. Cell. 93:165-176.

16. Hofbauer, L.C., and Heufelder, A.E. 2001. The role of osteoprotegerin and receptor activator of nuclear factor kappaB ligand in the pathogenesis and treatment of rheumatoid arthritis. Arthritis Rheum. 44:253-259.

17. Nakagawa, N., et al. 1998. RANK is the essential signaling receptor for osteoclast differentiation factor in osteoclastogenesis. Biochem. Biophys. Res. Commun. 253:395-400.

18. Simonet, W.S., et al. 1997. Osteoprotegerin: a novel secreted protein involved in the regulation of bone density. Cell. 89:309-319.

19. Ritchlin, C., et al. 1998. Patterns of cytokine production in psoriatic synovium. J. Rheumatol. 25:1544-1552.

20. Partsch, G., et al. 1998. T cell derived cytokines in psoriatic arthritis synovial fluids. Ann. Rheum. Dis. 57:691-693.

21. Danning, C.L., et al. 2000. Macrophage-derived cytokine and nuclear factor kappa B p65 expression in synovial membrane and skin of patients with psoriatic arthritis. Arthritis Rheum. 43:1244-1256.

22. Moll, J.M., and Wright, V. 1973. Psoriatic arthritis. Semin. Arthritis Rheum. 3:55-78.

23. Arnett, F.C., et al. 1988. The American Rheumatism Association 1987 revised criteria for the classification of rheumatoid arthritis. Arthritis Rheum. 31:315-324.

24. Shalhoub, V., et al. 2000. Characterization of osteoclast precursors in human blood. Br. J. Haematol. 111:501-512.

25. Ritchlin, C., and Haas-Smith, S.A. 2001. Expression of interleukin 10 mRNA and protein by synovial fibroblastoid cells. J. Rheumatol. 28:698-705.

26. Gravallese, E.M., et al. 2000. Synovial tissue in rheumatoid arthritis is a source of osteoclast differentiation factor. Arthritis Rheum. 43:250-258.

27. Myers, D.E., et al. 1999. Expression of functional RANK on mature rat and human osteoclasts. FEBS Lett. 463:295-300.

28. Huang, L., Xu, J., Wood, D.J., and Zheng, M.H. 2000. Gene expression of osteoprotegerin ligand, osteoprotegerin, and receptor activator of NF-kappaB in giant cell tumor of bone: possible involvement in tumor cell-induced osteoclast-like cell formation. Am. J. Pathol. 156:761-767.

29. Kong, Y.Y., et al. 1999. Activated T cells regulate bone loss and joint destruction in adjuvant arthritis through osteoprotegerin ligand. Neurosurgery. 402:304-309.

30. Pettit, A.R., et al. 2001. TRANCE/RANKL knockout mice are protected from bone erosion in a serum transfer model of arthritis. Am. J. Pathol. 159:1689-1699.

31. Redlich, K., et al. 2002. Tumor necrosis factor alpha-mediated joint 
destruction is inhibited by targeting osteoclasts with osteoprotegerin. Arthritis Rheum. 46:785-792.

32. Kobayashi, K., et al. 2000. Tumor necrosis factor alpha stimulates osteoclast differentiation by a mechanism independent of the ODF/RANKLRANK interaction. J. Exp. Med. 191:275-286.

33. Azuma, Y., Kaji, K., Katogi, R., Takeshita, S., and Kudo, A. 2000. Tumor necrosis factor-alpha induces differentiation of and bone resorption by osteoclasts. J. Biol. Chem. 275:4858-4864.

34. Martel, W., Hayes, J.T., and Duff, I.F. 1965. The pattern of bone erosion in the hand and wrist in rheumatoid arthrits. Radiology. 84:204-208.

35. Faust, J., et al. 1999. Osteoclast markers accumulate on cells developing from human peripheral blood mononuclear precursors. J. Cell. Biochem. 72:67-80.

36. Mease, P.J., et al. 2000. Etanercept in the treatment of psoriatic arthritis and psoriasis: a randomised trial. Lancet. 356:385-390.

37. Mease, P.J., Goffe, B., Metz, J., and Vanderstoep, A. 1999. Embrel (etanercept) in patients with psoriatic arthritis and psoriasis. Arthritis Rheum. 42:377. (Abstr.)

38. Marzo-Ortega, H., McGonagle, D., O’Connor, P., and Emery, P. 2001. Efficacy of etanercept in the treatment of the entheseal pathology in resistant spondylarthropathy: a clinical and magnetic resonance imaging study. Arthritis Rheum. 44:2112-2117.

39. Fujikawa, Y., Sabokbar, A., Neale, S., and Athanasou, N.A. 1996. Human osteoclast formation and bone resorption by monocytes and synovial macrophages in rheumatoid arthritis. Ann. Rheum. Dis. 55:816-822.

40. Quinn, J.M., Elliott, J., Gillespie, M.T., and Martin, T.J. 1998. A combination of osteoclast differentiation factor and macrophage-colony stimulating factor is sufficient for both human and mouse osteoclast formation in vitro. Endocrinology. 139:4424-4427.

41. Kotake, S., et al. 2001. Activated human T cells directly induce osteoclastogenesis from human monocytes: possible role of $\mathrm{T}$ cells in bone destruction in rheumatoid arthritis patients. Arthritis Rheum. 44:1003-1012.

42. Nicholson, G.C., et al. 2000. Induction of osteoclasts from CD14-positive human peripheral blood mononuclear cells by receptor activator of nuclear factor kappaB ligand (RANKL). Clin. Sci. 99:133-140.
43. Gori, F., et al. 2000. The expression of osteoprotegerin and RANK ligand and the support of osteoclast formation by stromal-osteoblast lineage cells is developmentally regulated. Endocrinology. 141:4768-4776.

44. Gravallese, E.M., and Goldring, S.R. 2000. Mechanisms of bone loss in inflammatory arthritis: diagnosis and therapeutic implications. Arthritis Res. 2:33-37.

45. Shigeyama, Y., et al. 2000. Expression of osteoclast differentiation factor in rheumatoid arthritis. Arthritis Rheum. 43:2523-2530.

46. Takayanagi, H., et al. 2000. T-cell-mediated regulation of osteoclastogenesis by signalling cross-talk between RANKL and IFN-gamma. Neurosurgery. 408:600-605.

47. Takayanagi, H., et al. 1997. A new mechanism of bone destruction in rheumatoid arthritis: synovial fibroblasts induce osteoclastogenesis. Biochem. Biophys. Res. Commun. 240:279-286.

48. Gravallese, E.M., et al. 1998. Identification of cell types responsible for bone resorption in rheumatoid arthritis and juvenile rheumatoid arthritis. Am. J. Pathol. 152:943-951.

49. Toritsuka, Y., et al. 1997. Osteoclastogenesis in iliac bone marrow of patients with rheumatoid arthritis. J. Rheumatol. 24:1690-1696.

50. Bromley, M., and Woolley, D.E. 1984. Chondroclasts and osteoclasts at subchondral sites of erosion in the rheumatoid joint. Arthritis Rheum. 27:968-975.

51. Reece, R.J., Canete, J.D., Parsons, W.J., Emery, P., and Veale, D.J. 1999. Distinct vascular patterns of early synovitis in psoriatic, reactive, and rheumatoid arthritis. Arthritis Rheum. 42:1481-1484.

52. Collin-Osdoby, P., et al. 2001. Receptor activator of NF-kappa B and osteoprotegerin expression by human microvascular endothelial cells, regulation by inflammatory cytokines, and role in human osteoclastogenesis. J. Biol. Chem. 276:20659-20672.

53. McGowan, N.W., Walker, E.J., Macpherson, H., Ralston, S.H., and Helfrich, M.H. 2001. Cytokine-activated endothelium recruits osteoclast precursors. Endocrinology. 142:1678-1681.

54. Hofbauer, L.C., and Heufelder, A.E. 2000. The role of receptor activator of nuclear factor-kappa B ligand and osteoprotegerin in the pathogenesis and treatment of metabolic bone diseases. J. Clin. Endocrinol. Metab. 85:2355-2363. 